\title{
Holomorphic Sectional Curvature of Complex Finsler Manifolds
}

\author{
Xueyuan Wan ${ }^{1}$
}

Received: 5 May 2017 / Published online: 25 January 2018

(C) The Author(s) 2018. This article is an open access publication

\begin{abstract}
In this paper, we get an inequality in terms of holomorphic sectional curvature of complex Finsler metrics. As applications, we prove a Schwarz Lemma from a complete Riemannian manifold to a complex Finsler manifold. We also show that a strongly pseudoconvex complex Finsler manifold with semi-positive but not identically zero holomorphic sectional curvature has negative Kodaira dimension under an extra condition.
\end{abstract}

Keywords Holomorphic sectional curvature · Complex Finsler manifolds ·

Schwarz Lemma · Kodaira dimension

Mathematics Subject Classification 53C60 - 53C55

\section{Introduction}

In this paper, we study the holomorphic sectional curvature of complex Finsler manifolds (see Definition 2.3). For a general complex manifold, there is a natural and intrinsic Finsler pseudo-metric, i.e., Kobayashi metric $k_{M}$ (see Definition 3.6), which is the maximum pseudo-metric among the pseudo metrics satisfying the decreasing property. This metric defines a Kobayashi pseudo-distance, and a complex manifold is called Kobayashi hyperbolic if the Kobayashi pseudo-distance is a distance in com-

Partially supported by NSFC (Grant Nos. 11221091, 11571184).

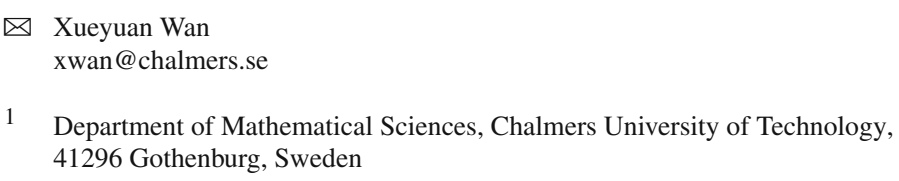


mon sense. It is known that a complex Finsler manifold is Kobayashi hyperbolic if its holomorphic sectional curvature is bounded from above by a negative constant. As an application, the moduli space of canonically polarized complex manifolds is Kobayashi hyperbolic [23,25].

In hyperbolic geometry a conjecture of Kobayashi asserts that the canonical bundle is ample if the manifold is hyperbolic [19, p. 370]. Wu and Yau [28] proved the ampleness of the canonical bundle for a projective manifold admitting a Kähler metric with negative holomorphic sectional curvature. Later on, Tosatti and Yang [26] proved this result without projective condition and asked if it still true for a compact Hermitian manifold. In [33], Yau conjectured that an algebraic manifold is of general type if and only if it admits a complex Finsler metric with strongly negative holomorphic sectional curvature which may be degenerate along a subvariety. For the case of quasi-negative holomorphic sectional curvature, the ampleness of the canonical bundle also had been proved in $[12,29]$. In the proofs of above results, they essentially used Yau's Schwarz Lemma [31]. This is also a motivation for us to study the Schwarz Lemma in the case of complex Finsler manifolds.

Proposition 1.1 Let $M$ be a complex manifold of dimension $n, G_{1}$ and $G_{2}$ be two complex Finsler metrics on $M$. For any point $(z,[v]) \in P(T M)$, one has

$$
\frac{2}{G_{2}} \sup _{\pi_{*}(X)=v}\left\{\partial \bar{\partial} \log \frac{G_{1}}{G_{2}}(X, \bar{X})\right\} \geq K_{G_{2}}-\frac{G_{1}}{G_{2}} K_{G_{1}},
$$

where $\pi_{*}: T(T M) \rightarrow T M$ is the differential of $\pi: T M \rightarrow M$.

A simple maximum principle argument immediately gives a direct proof for the Schwarz Lemma of [24, Theorem 4.1]. For the case of a complete Riemann surface $\left(M, G_{1}\right)$, by using Proposition 1.1 , we obtain the following generalization of Yau's Schwarz Lemma [31, Theorem 2'].

Theorem 1.2 Let $\left(M, G_{1}\right)$ be a complete Riemann surface with curvature bounded from below by a constant $K_{1}$. Let $\left(N, G_{2}\right)$ be another Finsler manifold with holomorphic sectional curvature bounded from above by a negative constant $K_{2}$. Then for any holomorphic map $f$ from $M$ to $N$,

$$
\frac{f^{*} G_{2}}{G_{1}} \leq \frac{K_{1}}{K_{2}}
$$

If $\left(M, G_{1}\right)$ is a unit disc with a Poincaré metric (which is complete) and by the definition of Kobayashi metric, one has $4 k_{N}^{2} \geq-K_{2} G_{1}$. This implies that $N$ is Kobayashi hyperbolic by definition.

For a compact Kähler manifold with positive holomorphic sectional curvature, Yau [32, Problem 67] asked if the manifold has negative Kodaira dimension. Yang [34] gave a affirmative answer to this problem. More precisely, if $(M, \omega)$ is a Hermitian manifold with semi-positive but not identically zero holomorphic sectional curvature, then the Kodaira dimension $\kappa(M)=-\infty$. Naturally, one may ask whether this result holds for a strongly pseudoconvex complex Finsler manifold, namely, whether for a 
compact strongly pseudoconvex Finsler manifold $(M, G)$ with semi-positive but not identically zero holomorphic sectional curvature one has that $\kappa(M)=-\infty$.

For any strongly pseudoconvex complex Finsler metric $G$, there is a canonical (Finslerian) tensor

$$
\hat{G}:=\frac{\partial^{2} G}{\partial v^{i} \partial v^{j}} \delta v^{i} \otimes \delta v^{j} \in A^{0}\left((T M)^{o}, \mathcal{V}^{*} \otimes \mathcal{V}^{*}\right)
$$

Here $(T M)^{o}$ denotes that the set of all non-zero holomorphic tangent vectors of $M,\left\{\delta v^{i}\right\}_{i=1}^{n}$ is a local holomorphic frame of $\mathcal{V}^{*}$ (for the definitions of $\delta v^{i}$ and $\mathcal{V}^{*}$ see (2.12)). By taking covariant derivative of the Finslerian tensor $\hat{G}$ along the antiholomorphic direction $\overline{\hat{P}}:=\overline{v^{i} \frac{\delta}{\delta z^{i}}}$, we get

$$
\bar{\partial} \hat{G}(\overline{\hat{P}}) \in A^{0}\left((T M)^{o}, \mathcal{V}^{*} \otimes \mathcal{V}^{*}\right) .
$$

By using Berndtsson's curvature formula to the cotangent bundle, we prove that

Theorem 1.3 Let $(M, G)$ be a compact strongly pseudoconvex complex Finsler manifold with semi-positive but not identically zero holomorphic sectional curvature, and satisfy $\bar{\partial} \hat{G}(\overline{\hat{P}})=0$, then $\kappa(M)=-\infty$.

Remark 1.4 From the definition of $\hat{P}$ and $\hat{G}$ (see $(2.15,2.25)$ in Sect. 2), if $G$ comes from a Hermitian metric, the Finslerian tensor $\hat{G}$ is identically trivial, so that any Hermitian metric satisfies the condition $\bar{\partial} \hat{G}(\overline{\hat{P}})=0$. Moreover, there are also many non-Hermitian strongly pseudoconvex complex Finsler metrics which satisfy the condition (see Example 2.6).

\section{Holomorphic Sectional Curvature of Complex Finsler Manifolds}

In this section, we shall fix notation and recall some basic definitions and facts on complex Finsler manifolds. For more details we refer to [1,4,5, 10, 13, 18,27].

Let $M$ be a complex manifold of dimension $n$, and let $\pi: T M \rightarrow M$ be the holomorphic tangent bundle of $M$. Let $z=\left(z^{1}, \ldots, z^{n}\right)$ be a local coordinate system in $M$, and let $\left\{\frac{\partial}{\partial z^{i}}\right\}_{1 \leq i \leq n}$ denote the corresponding natural frame of $T M$. So any element in $T M$ can be written as

$$
v=v^{i} \frac{\partial}{\partial z^{i}} \in T M
$$

where we adopt the summation convention of Einstein. In this way, one gets a local coordinate system on the complex manifold $T M$ :

$$
(z ; v)=\left(z^{1}, \ldots, z^{n} ; v^{1}, \ldots, v^{n}\right) .
$$

Definition 2.1 ([18,19]) A Finsler metric $G$ on the complex manifold $M$ is a continuous function $G: T M \rightarrow \mathbb{R}$ satisfying the following conditions: 
(F1): $G$ is smooth on $(T M)^{o}=T M \backslash O$, where $O$ denotes the zero section of $T M$;

(F2): $G(z, v) \geq 0$ for all $(z, v) \in T M$ with $z \in M$ and $v \in \pi^{-1}(z)$, and $G(z, v)=0$ if and only if $v=0$;

(F3): $G(z, \lambda v)=|\lambda|^{2} G(z, v)$ for all $\lambda \in \mathbb{C}$.

Moreover, $G$ is called strongly pseudoconvex if

(F4): the Levi form $\sqrt{-1} \partial \bar{\partial} G$ on $(T M)^{o}$ is positive-definite along fibers $(T M)_{z}=$ $\pi^{-1}(z)$ for $z \in M$.

$(M, G)$ is called a (strongly pseudoconvex) complex Finsler manifold if $G$ is a (strongly pseudoconvex) complex Finsler metric.

Clearly, any Hermitian metric on $M$ is naturally a strongly pseudoconvex complex Finsler metric on it.

We write

$$
\begin{aligned}
& G_{i}=\frac{\partial G}{\partial v^{i}}, \quad G_{\bar{j}}=\frac{\partial G}{\partial \bar{v}^{j}}, \quad G_{i \bar{j}}=\frac{\partial^{2} G}{\partial v^{i} \partial \bar{v}^{j}}, \\
& G_{i ; j}=\frac{\partial^{2} G}{\partial v^{i} \partial z^{j}}, \quad G_{i \bar{j} ; \bar{l}}=\frac{\partial^{3} G}{\partial v^{i} \partial \bar{v}^{j} \partial \bar{z}^{\beta}}, \quad \text { etc. }
\end{aligned}
$$

to denote the differentiation with respect to $v^{i}, \bar{v}^{j}, z^{i}, \bar{z}^{j}$, and we denote $\left(G^{\bar{j} i}\right)$ the inverse matrix of $\left(G_{i} \bar{j}\right)$. In the following lemma we collect some useful identities related to a Finsler metric $G$.

Lemma 2.2 ([10,18]) The following identities hold for any $(z, v) \in E^{o}, \lambda \in \mathbb{C}$ :

$$
\begin{aligned}
G_{i}(z, \lambda v) & =\bar{\lambda} G_{i}(z, v), \quad G_{i \bar{j}}(z, \lambda v)=G_{i \bar{j}}(z, v)=\bar{G}_{j \bar{i}}(z, v) ; \\
G_{i}(z, v) v^{i} & =G_{\bar{j}}(z, v) \bar{v}^{j}=G_{i \bar{j}}(z, v) v^{i} \bar{v}^{j}=G(z, v) ; \\
G_{i j}(z, v) v^{i} & =G_{i \bar{j} k}(z, v) v^{i}=G_{i \bar{j} \bar{k}}(z, v) \bar{v}^{j}=0 .
\end{aligned}
$$

Let $\Delta=\{w \in \mathbb{C}|| w \mid<1\}$ be a unit disc. For any holomorphic map $\varphi: \Delta \rightarrow M$, one can define a conformal metric on the disc $\Delta$ by

$$
d s^{2}=\varphi^{*} G d w \otimes d \bar{w}
$$

where $\left(\varphi^{*} G\right)\left(u \frac{\partial}{\partial w}\right):=G\left(\varphi_{*}\left(u \frac{\partial}{\partial w}\right)\right)$. The Gaussian curvature $K_{\varphi^{*} G}$ of $\varphi^{*} G$ is given by

$$
K_{\varphi^{*} G}=-\frac{2}{\varphi^{*} G} \frac{\partial^{2} \log \varphi^{*} G}{\partial w \partial \bar{w}} .
$$

Then one can define the holomorphic sectional curvature of $(M, G)$ as follows.

Definition $2.3([2,4])$ The holomorphic sectional curvature $K_{G}(z,[v])$ of $G$ at $(z,[v]) \in P(T M):=(T M)^{o} / \mathbb{C}^{*}$ is defined by

$$
K_{G}(z,[v]):=\sup _{\varphi}\left\{K_{\varphi^{*} G}(0)\right\}
$$


where the supremum is taken over all the holomorphic maps $\varphi: \Delta \rightarrow M$ satisfying $\varphi(0)=z, \varphi^{\prime}(0)=\lambda v$ for some $\lambda \in \mathbb{C}^{*}$.

If $G$ is a strongly pseudoconvex complex Finsler metric on $M$, then there is a canonical h-v decomposition of the holomorphic tangent bundle $T(T M)^{o}$ of $(T M)^{o}$ (see $[10, \S 5]$ or $[13, \S 1])$.

$$
T(T M)^{o}=\mathcal{H} \oplus \mathcal{V}
$$

In terms of local coordinates,

$$
\begin{aligned}
& \mathcal{H}=\operatorname{span}_{\mathbb{C}}\left\{\frac{\delta}{\delta z^{i}}=\frac{\partial}{\partial z^{i}}-G_{\bar{j} ; i} G^{\bar{j} k} \frac{\partial}{\partial v^{k}}, 1 \leq i \leq n\right\}, \\
& \mathcal{V}=\operatorname{span}_{\mathbb{C}}\left\{\frac{\partial}{\partial v^{i}}, 1 \leq i \leq n\right\} .
\end{aligned}
$$

Moreover, the dual bundle $T^{*}(T M)^{o}$ also has a smooth h-v decomposition $T^{*}(T M)^{o}=\mathcal{H}^{*} \oplus \mathcal{V}^{*}$ with

$$
\begin{aligned}
& \mathcal{H}^{*}=\operatorname{span}_{\mathbb{C}}\left\{d z^{i}, 1 \leq i \leq n\right\}, \\
& \mathcal{V}^{*}=\operatorname{span}_{\mathbb{C}}\left\{\delta v^{i}=d v^{i}+G^{\bar{j} i} G_{\bar{j} ; k} d z^{k}, \quad 1 \leq i \leq n\right\} .
\end{aligned}
$$

With respect to the $\mathrm{h}-\mathrm{v}$ decomposition $(2.12)$, the $(1,1)$-form $\frac{\sqrt{-1}}{2 \pi} \partial \bar{\partial} \log G$ has the following decomposition.

Lemma 2.4 ([5,18]) Let $G$ be a strongly pseudoconvex complex Finsler metric on $M$. One has

$$
\frac{\sqrt{-1}}{2 \pi} \partial \bar{\partial} \log G=-\frac{\Psi}{2 \pi}+\omega_{F S}
$$

where we denote

$$
\Psi=\sqrt{-1} R_{i \bar{j} k \bar{l}} \frac{v^{i} \bar{v}^{j}}{G} d z^{k} \wedge d \bar{z}^{l}, \quad \omega_{F S}=\frac{\sqrt{-1}}{2 \pi} \frac{\partial^{2} \log G}{\partial v^{i} \partial \bar{v}^{j}} \delta v^{i} \wedge \delta \bar{v}^{j},
$$

with

$$
R_{i \bar{j} k \bar{l}}=-\frac{\partial^{2} G_{i \bar{j}}}{\partial z^{k} \partial \bar{z}^{l}}+G^{\bar{q} p} \frac{\partial G_{i \bar{q}}}{\partial z^{k}} \frac{\partial G_{p \bar{j}}}{\partial \bar{z}^{l}} .
$$

For the holomorphic vector bundle $T(T M)^{o} \rightarrow(T M)^{o}$, there are two special smooth vector fields

$$
\hat{P}=v^{i} \frac{\delta}{\delta z^{i}} \in \mathcal{H}, \quad P=v^{i} \frac{\partial}{\partial v^{i}} \in \mathcal{V},
$$


which are well defined. Indeed, for two local coordinate neighborhoods $\left(U_{\alpha}\right.$, $\left.\left\{z_{\alpha}^{i}\right\}\right),\left(U_{\beta},\left\{z_{\beta}^{j}\right\}\right)$ of $M$ with $U_{\alpha} \cap U_{\beta} \neq \emptyset$, one has

$$
v_{\alpha}^{i}=\frac{\partial z_{\alpha}^{i}}{\partial z_{\beta}^{j}} v_{\beta}^{j}, \quad \frac{\delta}{\delta z_{\alpha}^{i}}=\frac{\partial z_{\beta}^{j}}{\partial z_{\alpha}^{i}} \frac{\delta}{\delta z_{\beta}^{j}}
$$

For a strongly pseudoconvex complex Finsler metric $G$, one can also define the holomorphic sectional curvature by

$$
K_{G}=2 R_{i \bar{j} k \bar{l}} \frac{v^{i} \bar{v}^{j} v^{k} \bar{v}^{l}}{G^{2}}=-\frac{2}{G}(\partial \bar{\partial} \log G)(\hat{P}, \overline{\hat{P}}),
$$

(see [18, Formula (6.7)]), which is a function on projective bundle $P(T M)$. In this case, the two kinds of definitions for holomorphic sectional curvature coincide. More precisely, one has

Proposition 2.5 ([1, Corollary 2.5.4, 3, Proposition 7.2]) If G is a strongly pseudoconvex complex Finsler metric on $M$, then

$$
\sup _{\varphi}\left\{K_{\varphi^{*} G}(0)\right\}=2 R_{i \bar{j} k \bar{l}} \frac{v^{i} \bar{v}^{j} v^{k} \bar{v}^{l}}{G^{2}}
$$

where the supremum is taken over all the holomorphic maps $\varphi: \Delta \rightarrow M$ satisfying $\varphi(0)=z, \varphi^{\prime}(0)=\lambda v$ for some $\lambda \in \mathbb{C}^{*}$.

Proof For reader's convenient, we give a direct proof here. For any holomorphic $\varphi: \Delta \rightarrow M$ with $\varphi(0)=z, \varphi^{\prime}(0)=\lambda v$ for some $\lambda \in \mathbb{C}^{*}$, it induces a holomorphic map

$$
\varphi_{*}: T \Delta \rightarrow T M \quad \varphi_{*}(w, u)=\varphi_{*}\left(u \frac{\partial}{\partial w}\right)=u \frac{\partial \varphi^{i}}{\partial w} \frac{\partial}{\partial z^{i}}=\left(\varphi(z) ; u \frac{\partial \varphi^{i}}{\partial w}\right)
$$

which satisfies

$$
\varphi_{*}(0,1)=(z, \lambda v)
$$

Similarly, the holomorphic map $\varphi_{*}$ also induces a holomorphic map $\left(\varphi_{*}\right)_{*}: T(T \Delta) \rightarrow$ $T(T M)$ and

$$
\left(\varphi_{*}\right)_{*}\left(\left.\frac{\partial}{\partial w}\right|_{(0,1)}\right)=\left.\lambda v^{i} \frac{\partial}{\partial z^{i}}\right|_{(z, \lambda v)}+\left.\frac{\partial^{2} \varphi^{i}}{\partial w^{2}} \frac{\partial}{\partial v^{i}}\right|_{(z, \lambda v)}
$$


Therefore, one has

$$
\begin{aligned}
& \sup _{\varphi}\left\{K_{\varphi^{*} G}(0)\right\}=\sup _{\varphi}\left\{-\frac{2}{G(z, \lambda v)}\left(\frac{\partial^{2}}{\partial w \partial \bar{w}} \log \varphi^{*} G\right)(0)\right\} \\
& =\sup _{\varphi}\left\{-\frac{2}{G(z, \lambda v)}\left(\varphi_{*}\right)^{*}(\partial \bar{\partial} \log G)\left(\frac{\partial}{\partial w}\left|(0,1), \frac{\partial}{\partial \bar{w}}\right|_{(0,1)}\right)\right\} \\
& =\sup _{\varphi}\left\{-\frac{2}{G(z, \lambda v)}(\partial \bar{\partial} \log G)\right. \\
& \left.\times\left(\left(\varphi_{*}\right)_{*}\left(\left.\frac{\partial}{\partial w}\right|_{(0,1)}\right),\left(\varphi_{*}\right)_{*}\left(\left.\frac{\partial}{\partial \bar{w}}\right|_{(0,1)}\right)\right)\right\} \\
& =\sup _{\varphi}\left\{-\frac{2}{G(z, \lambda v)}(\partial \bar{\partial} \log G)\right. \\
& \times\left(\lambda v^{i} \frac{\delta}{\delta v^{i}}+\left(\frac{\partial^{2} \varphi^{k}}{\partial w^{2}}+G_{\bar{l} ; i} G^{\bar{l} k} \lambda v^{i}\right) \frac{\partial}{\partial v^{k}},\right.
\end{aligned}
$$

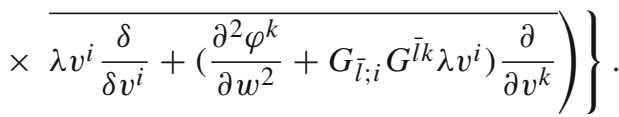

By Lemma 2.4 and the property that $\omega_{F S}$ is positive along the fiber of $P(T M)$, taking the holomorphic map $\varphi: \Delta \rightarrow M$ with

$$
\varphi(0)=0, \quad \varphi^{\prime}(0)=\lambda v, \quad \frac{\partial^{2} \varphi^{k}}{\partial w^{2}}(0)=-\lambda G_{\bar{l} ; i} G^{\bar{l} k} v^{i}
$$

one gets that

$$
\sup _{\varphi}\left\{K_{\varphi^{*} G}(0)\right\}=-\frac{2}{G(z, v)} \partial \bar{\partial} \log G\left(v^{i} \frac{\delta}{\delta v^{i}}, \overline{v^{i} \frac{\delta}{\delta v^{i}}}\right)=2 R_{i \bar{j} k \bar{l}} \frac{v^{i} \bar{v}^{j} v^{k} \bar{v}^{l}}{G^{2}}
$$

For a given strongly pseudoconvex complex Finsler metric $G$, there is a canonical (Finslerian) tensor $\hat{G}$,

$$
\hat{G}:=G_{i j} \delta v^{i} \otimes \delta v^{j} \in A^{0}\left((T M)^{o}, \mathcal{V}^{*} \otimes \mathcal{V}^{*}\right)
$$

From (2.16), it is easy to see that $\hat{G}$ is well defined. Moreover, $\hat{G}=0$ if and only if the strongly pseudoconvex complex Finsler metric $G$ comes from a Hermitian metric. In fact, if $G$ comes from Hermitian metric $h$ on $M$, i.e., $G=h_{i}(z) v^{i} \bar{v}^{j}$, then

$$
G_{i j}=\frac{\partial^{2} G}{\partial v^{i} \partial v^{j}}=0
$$

So $\hat{G}=0$. Conversely, if $\hat{G}=0$, then 


$$
G_{i \bar{k} j}=\frac{\partial^{2} G_{i j}}{\partial \bar{v}^{k}}=0
$$

By taking conjugation, one gets $G_{i \bar{k} \bar{j}}=0$. So $G_{i \bar{j}}(z, v)$ is independent of the fiber, i.e., $G_{i \bar{j}}(z, v)=G_{i \bar{j}}(z)$. Therefore, $G=G_{i \bar{j}}(z) v^{i} \bar{v}^{j}$ comes from a Hermitian metric.

Note that $\mathcal{V}^{*} \otimes \mathcal{V}^{*} \rightarrow(T M)^{o}$ is a holomorphic vector bundle with a local holomorphic frame $\left\{\delta v^{i} \otimes \delta v^{j}\right\}_{1 \leq i, j \leq n}$. So

$$
\bar{\partial} \hat{G} \in A^{0,1}\left((T M)^{o}, \mathcal{V}^{*} \otimes \mathcal{V}^{*}\right) .
$$

In applications, one may consider a class of strongly pseudoconvex complex Finsler metrics $G$, which satisfies

$$
\bar{\partial} \hat{G}(\overline{\hat{P}})=0 .
$$

In terms of local coordinates, Eq. (2.27) is equivalent to

$$
\bar{v}^{l} \frac{\delta}{\delta \bar{z}^{l}} G_{i j}=0 .
$$

Example 2.6 There are many non-Hermitian strongly pseudoconvex complex Finsler metrics which satisfy condition (2.27). For example, any complex Finsler manifold $(M, G)$ modeled on a complex Minkowski space satisfies the condition (2.27). In fact, in this case, one has $\frac{\partial}{\partial \bar{v}^{j}}\left(G_{\bar{l} ; i} G^{\bar{l} k}\right)=0$ (see [4, Definition 2.7, Proposition 2.10] or [15]). Then

$$
\begin{aligned}
\bar{v}^{l} \frac{\delta}{\delta \bar{z}^{l}} G_{i j} & =\bar{v}^{l}\left(G_{i j ; \bar{l}}-G_{k ; \bar{l}} G^{k \bar{t}} G_{i j \bar{t}}\right) \\
& =\bar{v}^{l} \frac{\partial}{\partial v^{j}}\left(G_{k ; \bar{l}} G^{\bar{t} k}\right) G_{i \bar{t}} \\
& =\bar{v}^{l} \frac{\partial}{\partial \bar{v}^{j}}\left(G_{\bar{k} ; l} G^{\bar{k} t}\right) G_{i \bar{t}}=0 .
\end{aligned}
$$

In particular, any flat or projectively flat Finsler metric $G$ verifies that $\frac{\partial}{\partial \bar{v}^{j}}\left(G_{\bar{l}_{; j}} G^{\bar{l} k}\right)=0$ (see [4, Corollary 2.2, Proposition 2.18]), and so one has $\bar{\partial} \hat{G}(\overline{\hat{P}})=0$.

\section{Schwarz Lemma for Complex Finsler Manifolds}

In this section, we get an inequality for complex Finsler manifolds which only contains holomorphic sectional curvature terms. By using this inequality and the Almost Maximum Principle of Omori and Yau for complete manifolds, we obtain a generalization of the Yau's Schwarz Lemma. 
Proposition 3.1 Let $M$ be a complex manifold of dimension n. Let $G_{1}$ and $G_{2}$ be two complex Finsler metrics on $M$. For any point $(z,[v]) \in P(T M)$, then

$$
\frac{2}{G_{2}} \sup _{\pi_{*}(X)=v}\left\{\partial \bar{\partial} \log \frac{G_{1}}{G_{2}}(X, \bar{X})\right\} \geq K_{G_{2}}-\frac{G_{1}}{G_{2}} K_{G_{1}},
$$

where $\pi_{*}: T(T M) \rightarrow T M$ is the differential of $\pi: T M \rightarrow M$.

Proof From Definition 2.3, one has at the point $(z,[v]) \in P(T M)$,

$$
\begin{aligned}
& \left(K_{G_{2}}-\frac{G_{1}}{G_{2}} K_{G_{1}}\right)(z,[v]) \\
& =\sup _{\varphi}\left\{K_{\varphi^{*} G_{2}}(0)\right\}-\frac{G_{1}(v)}{G_{2}(v)} \sup _{\varphi}\left\{K_{\varphi^{*} G_{1}}(0)\right\} \\
& =\sup _{\varphi}\left\{K_{\varphi^{*} G_{2}}(0)\right\}-\sup _{\varphi}\left\{\frac{G_{1}(v)}{G_{2}(v)} K_{\varphi^{*} G_{1}}(0)\right\} \\
& \leq \sup _{\varphi}\left\{K_{\varphi^{*} G_{2}}(0)-\frac{G_{1}(v)}{G_{2}(v)} K_{\varphi^{*} G_{1}}(0)\right\} \\
& =\sup _{\varphi}\left\{\frac{2}{\varphi^{*} G_{2}} \frac{\partial^{2}}{\partial w \partial \bar{w}} \log \frac{\varphi^{*} G_{1}}{\varphi^{*} G_{2}}\right\} \\
& =\sup _{\varphi}\left\{\frac{2}{G_{2}(\lambda v)} \partial \bar{\partial} \log \frac{G_{1}}{G_{2}}\left(\left(\varphi_{*}\right)_{*}\left(\frac{\partial}{\partial w}\right),\left(\varphi_{*}\right)_{*}\left(\frac{\partial}{\partial w}\right)\right)\right\} \\
& \leq \frac{2}{G_{2}(v)} \sup _{\pi_{*}(X)=v}\left\{\partial \bar{\partial} \log \frac{G_{1}}{G_{2}}(X, \bar{X})\right\},
\end{aligned}
$$

where the supremum is taken over all the holomorphic map $\varphi: \Delta \rightarrow M$ with $\varphi(0)=z$, $\varphi^{\prime}(0)=\lambda v$ for some $\lambda \in \mathbb{C}^{*}$.

Remark 3.2 From the proof of above proposition, if $G_{1}$ and $G_{2}$ only satisfy $(\mathbf{F 1}),(\mathbf{F 3})$, and non-negative, then (3.1) also holds outside the zero points of $G_{1} G_{2}$.

Remark 3.3 If $G_{1}$ and $G_{2}$ are strongly pseudoconvex complex Finsler metrics on $M$, then there is another upper-bound for $K_{G_{2}}-\frac{G_{1}}{G_{2}} K_{G_{1}}$. Denote by $\left(\frac{\delta}{\delta z^{i}}\right)_{\alpha}$ the horizontal lifting of $\frac{\partial}{\partial z^{i}}$ with respect to $G_{\alpha}, \alpha=1,2$. For any smooth function $f$ on $T M$, the horizontal Laplacian is defined by

$$
\Delta_{G_{2}}^{\mathcal{H}} f:=\frac{v^{i} \bar{v}^{j}}{G_{2}}(\partial \bar{\partial} f)\left(\left(\frac{\delta}{\delta z^{i}}\right)_{2},\left(\frac{\delta}{\delta \bar{z}^{j}}\right)_{2}\right) .
$$

From (2.17), one has 


$$
\begin{aligned}
2 \Delta_{G_{2}}^{\mathcal{H}} \log \frac{G_{1}}{G_{2}} & =K_{G_{2}}+\frac{2}{G_{2}}\left(\partial \bar{\partial} \log G_{1}\right)\left(\frac{\left.v^{i}\left(\frac{\delta}{\delta z^{i}}\right)_{2}, \overline{v^{i}\left(\frac{\delta}{\delta z^{i}}\right)_{2}}\right)}{v^{i}\left(\frac{\delta}{\delta z^{i}}\right)_{1}+v^{i}\left(\left(\frac{\delta}{\delta z^{i}}\right)_{2}-\left(\frac{\delta}{\delta z^{i}}\right)_{1}\right)}\right) \\
= & \left.K_{G_{2}}-\frac{G_{1}}{G_{2}} K_{G_{1}}+\frac{2}{G_{2}}\left(\frac{\delta}{\partial}\right)_{2}-\left(\frac{\delta}{\delta z^{i}}\right)_{1}\right), \\
& \times\left(v^{i}\left(\left(\frac{\delta}{\delta z^{i}}\right)_{2}-\left(\frac{\delta}{\delta z^{i}}\right)_{1}\right), \overline{v^{i}\left(\left(\frac{\delta}{\delta z^{i}}\right)_{2}-\left(\frac{\delta}{\delta z^{i}}\right)_{1}\right)}\right) \\
\geq & K_{G_{2}}-\frac{G_{1}}{G_{2}} K_{G_{1}},
\end{aligned}
$$

where the last inequality holds since $G_{1}$ is strongly pseudoconvex.

As an application of the above proposition, we give a direct proof of the following Schwarz Lemma.

Corollary 3.4 ([24, Theorem 4.1]) Let $f$ be a holomorphic map between two complex Finsler manifolds $(M, G)$ and $\left(N, G_{1}\right)$ with $M$ compact. If $K_{G_{1}} \leq-A, K_{G} \geq-B$ for $A>0, B \geq 0$, then

$$
f^{*} G_{1} \leq \frac{B}{A} G
$$

Proof Set

$$
u=\frac{f^{*} G_{1}}{G}
$$

which is a smooth function on $P(T M)$. If $\max _{(z,[v]) \in P(T M)} u(z,[v])=0$, then (3.3) holds obviously. Now we assume that

$$
\max _{(z,[v]) \in P(T M)} u(z,[v])=u\left(z_{0},\left[v_{0}\right]\right)>0 .
$$

By Remark 3.2, one has

$$
0 \geq \frac{2}{G\left(v_{0}\right)} \sup _{\pi_{*}(X)=v_{0}}\{\partial \bar{\partial} \log u(X, \bar{X})\} \geq\left(K_{G}-u K_{f^{*} G_{1}}\right)\left(z_{0},\left[v_{0}\right]\right) .
$$

Note that

$$
\begin{aligned}
K_{f^{*} G_{1}}([v]) & =\sup _{\varphi^{\prime}=\lambda v}\left\{K_{\varphi^{*} f^{*} G_{1}}(0)\right\} \leq \sup _{\psi^{\prime}=\lambda f_{*}(v)}\left\{K_{\psi^{*} G_{1}}(0)\right\} \\
& =K_{G_{1}}\left(\left[f_{*}(v)\right]\right)=\left(f^{*} G_{1}\right)[v] .
\end{aligned}
$$


So

$$
\left(K_{G}-u f^{*} K_{G_{1}}\right)\left(z_{0},\left[v_{0}\right]\right) \leq 0 .
$$

By the assumptions for $K_{G_{1}}$ and $K_{G_{2}}$, one obtains

$$
f^{*} G_{1} \leq \frac{B}{A} G
$$

Next we consider the case of a holomorphic map from a complete Riemann surface to a Finsler manifold with negative holomorphic sectional curvature. We assume that $(M, G)$ is a complete Riemann surface, the fundamental form is

$$
\Phi=\sqrt{-1} \lambda d z \wedge d \bar{z}, \quad \lambda=\frac{1}{2} G_{1 \overline{1}}
$$

and $d s^{2}=2 \lambda d z \otimes d \bar{z}$. The holomorphic sectional curvature of $G$ is

$$
K_{G}=-\frac{1}{\lambda} \frac{\partial^{2}}{\partial z \partial \bar{z}} \log \lambda,
$$

which is exactly the Gaussian curvature.

Now we have the following generalization of Yau's Schwarz Lemma [31, Theorem 2']

Theorem 3.5 Let $(M, G)$ be a complete Riemann surface with curvature bounded from below by a constant $K_{1}$. Let $\left(N, G_{1}\right)$ be another Finsler manifold with holomorphic sectional curvature bounded from above by a negative constant $K_{2}$. Then for any holomorphic map $f$ from $M$ to $N$,

$$
\frac{f^{*} G_{1}}{G} \leq \frac{K_{1}}{K_{2}}
$$

Proof Set

$$
u=\frac{f^{*} G_{1}}{G} .
$$

Since $\operatorname{dim} M=1$, one has that $P(T M) \simeq M$ and hence $u$ is a smooth function on $M$. For the case of $\max _{z \in M} u(z)=0$, the inequality (3.10) is obvious. So one may assume that $\max _{z \in M} u(z)=u\left(z_{0}\right)>0$ for some $z_{0} \in M$.

Let $\varphi(t)=(1+t)^{-1 / 2}, t>0$, then

$$
\varphi^{\prime}(t)=-\frac{1}{2(1+t)^{\frac{3}{2}}}<0, \quad \varphi^{\prime \prime}(t)=\frac{3}{4(1+t)^{\frac{5}{2}}}>0 .
$$

Then applying the Almost Maximum Principle of Omori and Yau ([22,30], [16, §6.2]) to $-\varphi \circ u$, there exists a sequence $\left\{p_{v} \in M \mid v=1,2, \ldots\right\}$ such that 


$$
\inf _{M} \varphi \circ u=\lim _{\nu \rightarrow \infty} \varphi \circ u\left(p_{v}\right),\left.\quad \lim _{\nu \rightarrow \infty} \nabla(\varphi \circ u)\right|_{p_{v}}=0,\left.\quad \liminf _{\nu \rightarrow \infty} \Delta(\varphi \circ u)\right|_{p_{v}} \geq 0 .
$$

The first equation of (3.12) implies that

$$
\sup _{M} u=\lim _{\nu \rightarrow \infty} u\left(p_{v}\right)
$$

From (3.5) and Proposition 3.1, one has

$$
\begin{aligned}
\Delta u & =\frac{2}{\lambda} \frac{\partial^{2} u}{\partial z \partial \bar{z}}=\frac{2}{\lambda}\left(u \frac{\partial^{2} \log u}{\partial z \partial \bar{z}}+\frac{1}{u}\left|\frac{\partial u}{\partial z}\right|^{2}\right) \\
& \geq 2 u\left(K_{G}-u K_{f^{*} G_{1}}\right) \geq 2 u\left(K_{G}-u f^{*} K_{G_{1}}\right) \\
& \geq 2 u\left(K_{1}-u K_{2}\right) .
\end{aligned}
$$

Using the fact that

$$
\left.\liminf _{\nu \rightarrow \infty} \Delta(\varphi \circ u)\right|_{p_{v}}=\liminf _{\nu \rightarrow \infty}\left(\varphi^{\prime \prime}\left(u\left(p_{\nu}\right)\right)\left\|\left.\nabla u\right|_{p_{v}}\right\|^{2}+\left.\varphi^{\prime}\left(u\left(p_{v}\right)\right) \Delta u\right|_{p_{v}}\right),
$$

and combining (3.12), (3.14), and $\varphi^{\prime}(t)<0$, one gets for any $\epsilon>0$, there exists $N>0$ such that at each $p_{v}$ with $v \geq N$,

$$
2 u\left(K_{1}-u K_{2}\right) \varphi^{\prime}(u)+\varphi^{\prime \prime}(u)\|\nabla u\|^{2}>-\epsilon, \quad\left(\varphi^{\prime}(u)\right)\|\nabla u\|^{2}=\|\nabla(\varphi \circ u)\|^{2}<\epsilon^{2} .
$$

Therefore,

$$
K_{1}-u K_{2}<\frac{1}{2 u}\left(\frac{\epsilon}{\left|\varphi^{\prime}(u)\right|}+\frac{\epsilon^{2} \varphi^{\prime \prime}(u)}{\left|\varphi^{\prime}(u)\right|^{3}}\right) .
$$

So $\sup _{M} u$ is bounded because the left-hand side is $O\left(u\left(p_{v}\right)\right)$ and right-hand side is $O\left(\epsilon\left(u\left(p_{\nu}\right)\right)^{\frac{1}{2}}+\epsilon^{2} u\left(p_{\nu}\right)\right)$ as $v \rightarrow \infty$. Taking $v \rightarrow \infty$ and $\epsilon \rightarrow 0$, one gets

$$
\sup _{M} u \leq \frac{K_{1}}{K_{2}}
$$

For a complex manifold, there is a canonical Kobayashi metric $k_{M}$.

Definition 3.6 $([4,19])$ The Kobayashi metric $k_{M}: T M \rightarrow \mathbb{R}$ of a complex manifold $M$ is defined by

$$
k_{M}(z, v):=\inf _{\varphi}\left\{\frac{1}{r} ; \exists f \in \operatorname{Hom}(\Delta(r), M), f(0)=z, f^{\prime}(0)=v\right\}
$$


for any $(z, v) \in T M$, where the infinimum is taken for all holomorphic map $f$ from $\operatorname{disc} \Delta(r)$ of radius $r$ to $M$ with $f(0)=z$ and $f^{\prime}(0)=v$.

For any tangent vector $V$ on $M$, there exists a $(1,0)$-type vector $X$ such that $V=$ $X+\bar{X}=2 \operatorname{Re} X, k_{M}(V):=2 k_{M}(X)$. The Kobayashi pseudo-distance $d_{M}^{K}(p, q)$ is defined by

$$
d_{M}^{K}(p, q)=\inf _{c} \int_{0}^{1} k_{M}\left(c(t), c^{\prime}(t)\right) \mathrm{d} t
$$

where infinimum is taken all curve of $C^{1}$-class on $M$.

A complex manifold $M$ is said to be Kobayashi hyperbolic if the pseudo-distance $d_{M}^{K}$ is the distance in the strict sense.

As a simple application, we have the following interesting result.

Corollary 3.7 ([4,18]) Let $\left(N, G_{1}\right)$ be a complex Finsler manifold with holomorphic sectional curvature bounded from above by a negative constant $K_{2}$. Then we have

$$
4 k_{N}^{2} \geq-K_{2} G_{1}
$$

where $k_{N}$ is the Kobayashi metric of $N$. In particular, $N$ is Kobayashi hyperbolic.

Proof We equip the $r$-disc $\Delta(r)$ with the complete Poincaré metric $G=2 \lambda d z \otimes d \bar{z}$, $\lambda=\frac{r^{2}}{2\left(r^{2}-|z|^{2}\right)}$. It is well known that its Gaussian curvature is $K_{G}=-4<0$. By Theorem 3.5, one has

$$
f^{*} G_{1} \leq \frac{-4}{K_{2}} G
$$

Therefore,

$$
-K_{2} G_{1}\left(f(0), f^{\prime}(0)\right) \leq\left.\frac{4 r^{2}}{\left(r^{2}-|z|^{2}\right)^{2}}\right|_{z=0}=\frac{4}{r^{2}} .
$$

By the definition of $k_{N}$, one has

$$
4 k_{N}^{2} \geq-K_{2} G_{1}
$$

From (3.20) and $K_{2}<0, d_{N}^{K}$ is a distance in the strict sense. By Definition 3.6, $N$ is Kobayashi hyperbolic.

\section{Semi-positive Holomorphic Sectional Curvature}

In this section, we assume that the holomorphic sectional curvature of a strongly pseudoconvex complex Finsler manifold $(M, G)$ is semi-positive but not identically zero, i.e., $K_{G} \geq 0$ and there exists a point $\left(z_{0},\left[v_{0}\right]\right) \in P(T M)$ such that $K_{G}\left(z_{0},\left[v_{0}\right]\right)>0$. Firstly, we review Berndtsson's curvature formula of direct image bundles (cf. [7$9,20,21])$. 


\subsection{Curvature of Direct Image Bundles}

Let $\pi: \mathcal{X} \rightarrow M$ be a holomorphic fibration with compact fibers, $L$ a relative ample line bundle over $\mathcal{X}$, i.e., there exists a metric (weight) $\phi$ of $L$ such that $\left.\sqrt{-1} \partial \bar{\partial} \phi\right|_{\mathcal{X}_{z}}>0$ for any $z \in M, \mathcal{X}_{z}:=\pi^{-1}(z)$. We denote by $(z ; w)=\left(z^{1}, \ldots, z^{n} ; w^{1}, \ldots, w^{m}\right)$ a local admissible holomorphic coordinate system of $\mathcal{X}$ with $\pi(z ; w)=z$, where $m$ denotes the dimension of fibers. For any smooth function $\phi$ on $\mathcal{X}$, we denote

$$
\phi_{; i}=\frac{\partial \phi}{\partial z^{i}}, \quad \phi_{; \bar{j}}=\frac{\partial \phi}{\partial \bar{z}^{j}}, \quad \phi_{\alpha}=\frac{\partial \phi}{\partial w^{\alpha}}, \quad \phi_{\bar{\beta}}=\frac{\partial \phi}{\partial \bar{w}^{\beta}},
$$

where $1 \leq i, j \leq n, 1 \leq \alpha, \beta \leq m$.

For any smooth metric $\phi$ of $L$ with $\left.\sqrt{-1} \partial \bar{\partial} \phi\right|_{\mathcal{X}_{z}}>0$, set

$$
\frac{\delta}{\delta z^{i}}:=\frac{\partial}{\partial z^{i}}-\phi_{\bar{\beta} ; i} \phi^{\bar{\beta} \alpha} \frac{\partial}{\partial w^{\alpha}},
$$

where $\left(\phi^{\bar{\beta} \alpha}\right)$ is the inverse matrix of $\left(\phi_{\alpha \bar{\beta}}\right)$. By a routine computation, one shows easily that $\left\{\frac{\delta}{\delta z^{i}}\right\}_{1 \leq i \leq n}$ spans a well-defined horizontal subbundle of $T \mathcal{X}$. Let $\left\{d z^{i} ; \delta w^{\alpha}\right\}$ denote the dual frame of $\left\{\frac{\delta}{\delta z^{i}} ; \frac{\partial}{\partial w^{\alpha}}\right\}$. One has

$$
\delta w^{\alpha}=d w^{\alpha}+\phi^{\alpha \bar{\beta}} \phi_{\bar{\beta} ; i} d z^{i}
$$

Denote

$$
\partial^{V}=\frac{\partial}{\partial w^{\alpha}} \otimes \delta w^{\alpha}, \quad \partial^{H}=\frac{\delta}{\delta z^{i}} \otimes d z^{i} .
$$

Clearly, the operators $\partial^{V}$ and $\partial^{H}$ are well defined.

The geodesic curvature $c(\phi)$ of $\phi$ ([11, Definition 2.1]) is defined by

$$
c(\phi)=c(\phi)_{i \bar{j}} \sqrt{-1} d z^{i} \wedge d \bar{z}^{j}=\left(\phi_{; i \bar{j}}-\phi_{\bar{\beta} ; i} \phi^{\alpha \bar{\beta}} \phi_{\alpha ; \bar{j}}\right) \sqrt{-1} d z^{i} \wedge d \bar{z}^{j},
$$

which is clearly a horizontal real $(1,1)$-form on $\mathcal{X}$. By a direct computation, one has

$$
\sqrt{-1} \partial \bar{\partial} \phi=c(\phi)+\sqrt{-1} \phi_{\alpha \bar{\beta}} \delta w^{\alpha} \wedge \delta \bar{w}^{\beta}
$$

We consider the direct image sheaf

$$
E:=\pi_{*}\left(K_{\mathcal{X} / M}+L\right)
$$

Then $E$ is a holomorphic vector bundle. In fact, for any point $p \in M$, taking a local coordinate neighborhood $\left(U ;\left\{z^{i}\right\}\right)$ of $p$, then $\phi+\beta \sum_{i=1}^{n}\left|z^{i}\right|^{2}$ is a metric on the line bundle $\left.L \rightarrow \mathcal{X}\right|_{U}$, whose curvature is 


$$
\sqrt{-1} \partial \bar{\partial} \phi+\beta \sqrt{-1} \sum_{i=1}^{n} d z^{i} \wedge d \bar{z}^{i}
$$

By taking $\beta$ large enough, the curvature of $\phi+\beta \sum_{i=1}^{n}\left|z^{i}\right|^{2}$ is positive. By the same argument as in [7, §4, p. 542], there exists a local holomorphic frame for $E$. So $E$ is a holomorphic vector bundle.

Following Berndtsson (cf. [7-9]), we define the following $L^{2}$-metric on the direct image bundle $E:=\pi_{*}\left(K_{\mathcal{X} / M}+L\right)$. Let $\left\{u^{A}\right\}_{1 \leq A \leq \operatorname{rank} E}$ be a local frame of $E$. Set

$$
h^{A \bar{B}}=\left\langle u^{A}, u^{B}\right\rangle=\int_{\mathcal{X}_{z}} u^{A} \overline{u^{B}} e^{-\phi} .
$$

Note that $u^{A}$ can be written locally as

$$
u^{A}=f^{A} d w \otimes s_{L}
$$

where $s_{L}$ is a local holomorphic frame of $L$ with $e^{-\phi}=\left|s_{L}\right|^{2}$, and so locally

$$
u^{A} \overline{u^{B}} e^{-\phi}:=(\sqrt{-1})^{m^{2}} f^{A} \overline{f^{B}}\left|s_{L}\right|^{2} d w \wedge d \bar{w}=(\sqrt{-1})^{m^{2}} f^{A} \overline{f^{B}} e^{-\phi} d w \wedge d \bar{w}
$$

where $d w=d w^{1} \wedge \cdots \wedge d w^{m}$ is the fiber volume.

The following theorem actually was proved by Berndtsson [9, Theorem 1.2].

Theorem 4.1 ([9, Theorem 1.2]) Denote by $\Theta^{E}=\Theta^{A \bar{B}}{ }_{i \bar{j}} u_{A} \otimes \bar{u}_{B} \otimes d z^{i} \wedge d \bar{z}^{j}$ the Chern curvature of the $L^{2}$-metric (4.5) on $E$, where $\left\{u_{A} \in E^{*}\right\}$ is the dual frame of $\left\{u^{A}\right\}$. Then at the point $z \in M$,

$$
\Theta_{i \bar{j}}^{A \bar{B}}=\int_{\mathcal{X}_{z}} c(\phi)_{i \bar{j}} u^{A} \bar{u}^{B} e^{-\phi}+\left\langle\left(1+\square^{\prime}\right)^{-1} i_{\bar{\partial} V \frac{\delta}{\delta z^{i}}} u^{A}, i_{\bar{\partial} V} \frac{\delta}{\delta z^{j}} u^{B}\right\rangle .
$$

Here $\square^{\prime}=\nabla^{\prime} \nabla^{\prime *}+\nabla^{\prime *} \nabla$ denotes the Laplacian on $\mathcal{X}_{z}$ associated with the $(1,0)$-part of the Chern connection on $\left.L\right|_{\mathcal{X}_{z}}, \bar{\partial}^{V} \frac{\delta}{\delta z^{i}}=\frac{\partial\left(-\phi_{\bar{\beta} ; i} \phi^{\bar{\beta} \alpha}\right)}{\partial \bar{w}^{\gamma}} \delta \bar{w}^{\gamma} \otimes \frac{\partial}{\partial w^{\alpha}}$.

Moreover,

$$
\begin{aligned}
& \left\langle\left(1+\square^{\prime}\right)^{-1} i_{\bar{\partial} V \frac{\delta}{\delta z^{i}}} u^{A}, i_{\bar{\partial}} V \frac{\delta}{\delta z^{j}} u^{B}\right) a_{A}^{i} \overline{a_{B}^{j}} \\
& =\left\langle\left(1+\square^{\prime}\right)^{-1}\left(i_{\bar{\partial}} V \frac{\delta}{\delta z^{i}} u^{A} a_{A}^{i}\right),\left(i_{\bar{\partial}} V \frac{\delta}{\delta z^{j}} u^{B} a_{B}^{j}\right)\right\rangle \geq 0
\end{aligned}
$$

for any element $a=a_{A}^{i} u^{A} \otimes \frac{\partial}{\partial z^{i}} \in E \otimes T M$, and the equality holds if and only if

$$
\frac{\partial\left(\phi_{\bar{\beta} ; i} \phi^{\bar{\beta} \alpha}\right)}{\partial \bar{w}^{\gamma}} f^{A} a_{A}^{i}=0 .
$$


Proof For the proof of (4.7), one also can refer to the proof of [14, Theorem 3.1]. The equality of (4.8) holds if and only if

$$
i_{\bar{\partial} V \frac{\delta}{\delta z^{i}}} u^{A} a_{A}^{i}=0
$$

From (4.1) and (4.2), the above equation is equivalent to $\frac{\partial\left(\phi_{\bar{\beta} ; i} \phi^{\bar{\beta} \alpha}\right)}{\partial \bar{w}^{\gamma}} f^{A} a_{A}^{i}=0$.

\subsection{Application on Cotangent Bundles}

In this subsection, we will apply Theorem 4.1 to the cotangent bundle $E=T^{*} M$. In this case, $\mathcal{X}=P(T M)$,

$$
E=\pi_{*}\left(\mathcal{O}_{P(T M)}(1)\right)=\pi_{*}\left(L+K_{\mathcal{X} / M}\right)
$$

where

$$
L:=\mathcal{O}_{P(T M)}(1)-K_{\mathcal{X} / M}=(n+1) \mathcal{O}_{P(T M)}(1)+\pi^{*} \operatorname{det} T M
$$

(see [17, Proposition 2.2]). In this case, $\operatorname{rank} E=\operatorname{dim} M=n$, the dimension of fibers $m=\operatorname{dim} \mathcal{X}_{z}=n-1$ for any $z \in M$.

Recall that $(z ; v)$ is a local coordinate system of $T M$ with respect to the natural frame $\left\{\frac{\partial}{\partial z^{i}}\right\}_{i=1}^{n}$, it gives a local holomorphic frame of $\mathcal{O}_{P(T M)}(-1)$ by

$$
s_{\mathcal{O}_{P(T M)}(-1)}(z,[v])=\frac{1}{v^{k}} \sum_{i=1}^{n} v^{i} \pi^{*} \frac{\partial}{\partial z^{i}}
$$

on $U_{k}:=\left\{(z,[v]) \in P(T M) \mid v^{k} \neq 0\right\}$.

Moreover, the natural projection

$$
q:(T M)^{o} \rightarrow P(T M) \quad(z ; v) \mapsto(z ;[v]):=\left(z^{1}, \ldots, z^{n} ;\left[v^{1}, \ldots, v^{n}\right]\right)
$$

gives a local coordinate system of $P(T M)$ by

$$
(z ; w)=\left(z^{1}, \ldots, z^{n} ; \frac{v^{1}}{v^{k}}, \ldots, \frac{v^{k-1}}{v^{k}}, \frac{v^{k+1}}{v^{k}}, \ldots, \frac{v^{n}}{v^{k}}\right)
$$

on $U_{k}$.

Let $s_{\mathcal{O}_{P(T M)}}$ (1) denote the dual local holomorphic section of $s_{\mathcal{O}_{P(T M)}(-1)}$. By (4.10), there exist local frame $s_{L}$ of $L$ and local frame $s_{\pi^{*} \operatorname{det} T M}$ of $\pi^{*} \operatorname{det} T M$ on $U_{k}$ such that

$$
s_{\mathcal{O}_{P(T M)}(1)}=d w \otimes s_{L}, \quad s_{L}=s_{\mathcal{O}_{P(T M)}(-1)}^{\otimes-(n+1)} \otimes s_{\pi * \operatorname{det} T M} .
$$


Let $G$ be a strongly pseudoconvex complex Finsler metric on $M$, it induces a metric on $\mathcal{O}_{P(T M)}(-1)$ by

$$
\left|s_{\mathcal{O}_{P(T M)}(-1)}\right|^{2}=\frac{1}{\left|v^{k}\right|^{2}} G_{i \bar{j}}(z, v) v^{i} \bar{v}^{j}=\frac{G(z, v)}{\left|v^{k}\right|^{2}}
$$

on $U_{k}$. Let $g=\left(g_{i} \bar{j}\right)$ be a Hermitian metric on $M$. From (4.14), there exists a metric $\phi_{L}$ on $L$ by

$$
e^{-\phi_{L}}:=\left|s_{L}\right|^{2}:=\left|s_{\mathcal{O}_{P(T M)}(-1)}\right|^{-2(n+1)}\left|s_{\pi^{*} \operatorname{det} T M}\right|^{2}=\left|v^{k}\right|^{2(n+1)} G^{-(n+1)} \operatorname{det} g,
$$

where $\operatorname{det} g:=\operatorname{det}\left(g_{i}\right)$.

Lemma 4.2 On $U_{k}=\left\{(z,[v]) \in P(T M) \mid v^{k} \neq 0\right\}$, one has

$$
\operatorname{det}\left(\frac{\partial^{2} \log G}{\partial w^{\alpha} \partial \bar{w}^{\beta}}\right)=\frac{\left|v^{k}\right|^{2 n}}{G^{n}} \operatorname{det} G
$$

where $\operatorname{det} G:=\operatorname{det}\left(G_{i} \bar{j}\right)$.

Proof Without loss of generality, we may assume that $k=1$. For any point $p \in U_{1}$, one has from (4.13),

$$
q_{*}\left(\frac{\partial}{\partial v^{1}}\right)=-\sum_{i=2}^{n} \frac{v^{i}}{\left(v^{1}\right)^{2}} \frac{\partial}{\partial w^{i-1}}, \quad q_{*}\left(\frac{\partial}{\partial v^{k}}\right)=\frac{1}{v^{1}} \frac{\partial}{\partial w^{k-1}}, \quad k \geq 2
$$

Here $q_{*}: T(T M)^{o} \rightarrow T P(T M)$ denotes the differential of the natural projection $q:(T M)^{o} \rightarrow P(T M)$.

Denote

$T=v^{k} \frac{\partial}{\partial v^{k}}$ and

$\left\langle\frac{\partial}{\partial v^{i}}, \frac{\partial}{\partial v^{j}}\right\rangle:=\frac{1}{G} G_{i \bar{j}}$, one has $\langle T, T\rangle=1$. With respect to the basis $\left\{T, \frac{\partial}{\partial v^{2}}, \ldots, \frac{\partial}{\partial v^{n}}\right\}$, the matrix of $\langle\cdot, \cdot\rangle$ is

$B\left(\frac{1}{G} G_{i}\right) \bar{B}^{T}$,

where

$$
B=\left[\begin{array}{cccc}
v^{1} & v^{2} & \ldots & v^{n} \\
0 & 1 & \ldots & 0 \\
\vdots & \vdots & \ddots & \vdots \\
0 & 0 & \ldots & 1
\end{array}\right]
$$

Denote $\left(\frac{\partial}{\partial v^{k}}\right)^{\perp}=\frac{\partial}{\partial v^{k}}-\left\langle\frac{\partial}{\partial v^{k}}, T\right\rangle T$. 
For any $i, j \geq 2$, we have from (4.16)

$$
\begin{aligned}
\left\langle\left(\frac{\partial}{\partial v^{i}}\right)^{\perp},\left(\frac{\partial}{\partial v^{j}}\right)^{\perp}\right\rangle & =\frac{1}{G} G_{i \bar{j}}-\frac{1}{G^{2}} G_{i} G_{\bar{j}} \\
& =\frac{\partial^{2} \log G}{\partial v^{i} \partial \bar{v}^{j}}=\frac{1}{\left|v^{1}\right|^{2}} \frac{\partial^{2} \log G}{\partial w^{i} \partial \bar{w}^{j}} .
\end{aligned}
$$

So with respect to the basis $\left\{T,\left(\frac{\partial}{\partial \zeta^{2}}\right)^{\perp}, \ldots,\left(\frac{\partial}{\partial \zeta^{r}}\right)^{\perp}\right\}$, the matrix of $\langle\cdot, \cdot\rangle$ is

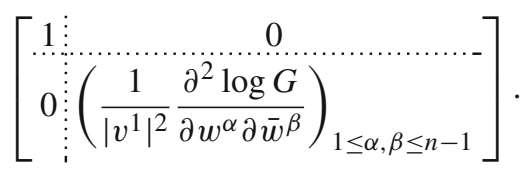

Since $\left\{T,\left(\frac{\partial}{\partial v^{2}}\right)^{\perp}, \ldots,\left(\frac{\partial}{\partial v^{n}}\right)^{\perp}\right\}$ and $\left\{T, \frac{\partial}{\partial v^{2}}, \ldots, \frac{\partial}{\partial v^{n}}\right\}$ differ by an unitary matrix, so

$$
\frac{1}{\left|v^{1}\right|^{2(n-1)}} \operatorname{det}\left(\frac{\partial^{2} \log G}{\partial w^{\alpha} \partial \bar{w}^{\beta}}\right)=\operatorname{det}\left(B\left(\frac{1}{G} G_{i \bar{j}}\right) \bar{B}^{T}\right)=\left|v^{1}\right|^{2} \frac{\operatorname{det}\left(G_{i \bar{j}}\right)}{G^{n}},
$$

which completes the proof.

Combining (4.15) with Lemma 4.2, one has

$$
e^{-\phi_{L}}=\frac{\left|v^{k}\right|^{2} \operatorname{det}\left(\partial_{w^{\alpha}} \partial_{\bar{w} \beta} \log G\right)}{G(\operatorname{det} G)(\operatorname{det} g)^{-1}}
$$

on $U_{k}$.

It is known that $H^{0}\left(\mathbb{P}^{n-1}, \mathcal{O}_{\mathbb{P}^{n-1}}(k)\right)$ can be identified as the space of homogeneous polynomials of degree $k$ in $n$ variables. Therefore, the sections of $H^{0}\left(\mathcal{X}_{z}, \mathcal{O}_{P(T M)}(1) \mid \mathcal{X}_{z}\right)$ are of the form

$$
u^{i}=v^{i}\left(v^{j} \pi^{*} \frac{\partial}{\partial z^{j}}\right)^{*}=\frac{v^{i}}{v^{k}} s_{\mathcal{O}_{P(T M)}(1)}=\frac{v^{i}}{v^{k}} d w \otimes s_{L} .
$$

On the other hand, there is a canonical element

$$
a:=u^{i} \otimes \frac{\partial}{\partial z^{i}}=\left(v^{i} \pi^{*} \frac{\partial}{\partial z^{i}}\right)^{*} \otimes v^{i} \frac{\partial}{\partial z^{i}} \in E \otimes T M,
$$

which is well defined since $v^{i}$ and $\frac{\partial}{\partial z^{i}}$ are covariant to each other.

By (4.18), (4.19), and Theorem 4.1, we obtain

$$
\begin{aligned}
\Theta_{i \bar{j}}^{i \bar{j}}= & \int_{\mathcal{X}_{z}} c(\phi)_{i} \frac{v^{i} \bar{v}^{j}}{\left|v^{k}\right|^{2}} e^{-\phi_{L}}(\sqrt{-1})^{(n-1)^{2}} d w \wedge d \bar{w} \\
& +\left\langle\left(1+\square^{\prime}\right)^{-1} i_{\bar{\partial} V \frac{\delta}{\delta z^{i}}} u^{i}, i_{\bar{\partial} V} \frac{\delta}{\delta z^{j}} u^{j}\right\rangle .
\end{aligned}
$$


Now let us compute the first term in the right-hand side of (4.20). By (4.15), (4.17), and setting $\phi_{G}=\log G$, one has

$$
\begin{aligned}
& \int_{\mathcal{X}_{z}} c\left(\phi_{L}\right)_{i} \bar{j} \frac{v^{i} \bar{v}^{j}}{\left|v^{k}\right|^{2}} e^{-\phi_{L}}(\sqrt{-1})^{(n-1)^{2}} d w \wedge d \bar{w} \\
& =\int_{\mathcal{X}_{z}}\left((n+1) c\left(\phi_{G}\right)_{i} \bar{j}-\partial_{i} \partial_{\bar{j}} \log \operatorname{det} g\right) \frac{v^{i} \bar{v}^{j}}{G} \frac{\left|v^{k}\right|^{2 n} \operatorname{det} g}{G^{n}}(\sqrt{-1})^{(n-1)^{2}} d w \wedge d \bar{w} \\
& =\int_{\mathcal{X}_{z}}\left((n+1) c\left(\phi_{G}\right)_{i} \bar{j}-\partial_{i} \partial_{\bar{j}} \log \operatorname{det} g\right) \frac{v^{i} \bar{v}^{j}}{G} \frac{\operatorname{det} g}{\operatorname{det} G} \frac{(\sqrt{-1} \partial \bar{\partial} \log G)^{n-1}}{(n-1) !} \\
& =-(n+1) \int_{\mathcal{X}_{z}} R_{i \bar{j} k \bar{l}} \frac{v^{i} \bar{v}^{j} v^{k} \bar{v}^{l}}{G^{2}} \frac{\operatorname{det} g}{\operatorname{det} G} \frac{(\sqrt{-1} \partial \bar{\partial} \log G)^{n-1}}{(n-1) !} \\
& \quad-\partial_{i} \partial_{\bar{j}} \log \operatorname{det} g \int_{\mathcal{X}_{z}} \frac{v^{i} \bar{v}^{j}}{G} \frac{\operatorname{det} g}{\operatorname{det} G} \frac{(\sqrt{-1} \partial \bar{\partial} \log G)^{n-1}}{(n-1) !} .
\end{aligned}
$$

Now the induced Hermitian metric on $T^{*} M$ is

$$
h^{\bar{j} i}=\int_{\mathcal{X}_{z}} \frac{v^{i} \bar{v}^{j}}{G} \frac{\operatorname{det} g}{\operatorname{det} G} \frac{(\sqrt{-1} \partial \bar{\partial} \log G)^{n-1}}{(n-1) !} .
$$

Denote by $h=\left(h_{i} \bar{j}\right)$ the dual Hermitian metric of $\left(h^{\bar{j} i}\right)$, and $\operatorname{det} h:=\left(\operatorname{det}\left(h^{\bar{j} i}\right)\right)^{-1}$. Consider the induced metric on $T^{*} M$ given by (4.22). We claim that by an appropriate rescaling of $g$ one can always reduce to the case in which $\operatorname{det} g=(\operatorname{det} h)^{-1}$. Indeed, in place of $g$ one can always consider the Hermitian metric

$$
\tilde{g}_{i \bar{j}}=\left(\frac{\operatorname{det} h}{\operatorname{det} g}\right)^{\frac{1}{n(n+1)}} g_{i \bar{j}}
$$

So there induces a Hermitian metric $\tilde{h}$ on $T^{*} M$ associated with the Hermitian metric $\tilde{g}=\left(\tilde{g}_{i}\right)$, which is given by (4.22). Moreover,

$$
\begin{aligned}
\operatorname{det}\left(\tilde{h}^{\overline{j i}}\right) & =\operatorname{det}\left(\int_{\mathcal{X}_{z}} \frac{v^{i} \bar{v}^{j}}{G} \frac{\operatorname{det} \tilde{g}}{\operatorname{det} G} \frac{(\sqrt{-1} \partial \bar{\partial} \log G)^{n-1}}{(n-1) !}\right) \\
& =\left(\frac{\operatorname{det} h}{\operatorname{det} g}\right)^{\frac{n}{n+1}}(\operatorname{det} h)^{-1} \\
& =\left(\left(\frac{\operatorname{det} h}{\operatorname{det} g}\right)^{\frac{1}{n+1}} \operatorname{det} g\right)^{-1} \\
& =(\operatorname{det} \tilde{g})^{-1} .
\end{aligned}
$$


For a Hermitian metric $h=\left(h_{i} \bar{j}\right)$ on $M$, the two scalar curvatures are defined by

$$
s_{h}=\Theta_{i \bar{j} k \bar{l}} h^{i \bar{j}} h^{k \bar{l}}, \quad \hat{s}_{h}=\Theta_{i \bar{j} k \bar{l}} h^{i \bar{l}} h^{k \bar{j}},
$$

where $\Theta_{i \bar{j} k \bar{l}} h^{i \bar{j}}=-\frac{\partial^{2} h_{k \bar{l}}}{\partial z^{i} \partial \bar{z}^{j}}+h^{\bar{q} p} \frac{\partial h_{p \bar{j}}}{\partial \bar{z}^{l}} \frac{\partial h_{i \bar{q}}}{\partial z^{k}}$.

From the chosen of Hermitian metric $g$ and (4.25), (4.21) reduces to

$$
\begin{aligned}
& \int_{\mathcal{X}_{z}} c\left(\phi_{L}\right)_{i} \bar{j} \frac{v^{i} \bar{v}^{j}}{\left|v^{k}\right|^{2}} e^{-\phi_{L}}(\sqrt{-1})^{(n-1)^{2}} d w \wedge d \bar{w} \\
& =-\frac{n+1}{2} \int_{\mathcal{X}_{z}} K_{G} \frac{\operatorname{det} g}{\operatorname{det} G} \frac{(\sqrt{-1} \partial \bar{\partial} \log G)^{n-1}}{(n-1) !}+s_{h} .
\end{aligned}
$$

Now we deal with the second term in the RHS of (4.20). By Theorem (4.1),

$$
\left\langle\left(1+\square^{\prime}\right)^{-1} i_{\bar{\partial} V \frac{\delta}{\delta z^{i}}} u^{i}, i_{\bar{\partial} V \frac{\delta}{\delta z^{j}}} u^{j}\right\rangle \geq 0
$$

and $\left\langle\left(1+\square^{\prime}\right)^{-1} i_{\bar{\partial} V} \frac{\delta}{\delta z^{i}} u^{i}, i_{\bar{\partial} V} \frac{\delta}{\delta z^{j}} u^{j}\right\rangle=0$ if and only if

$$
\frac{\partial\left(\left(\phi_{L}\right)_{\bar{\beta} ; i}\left(\phi_{L}\right)^{\bar{\beta} \alpha}\right)}{\partial \bar{w}^{\gamma}} v^{i}=0 .
$$

From the definition of $\phi_{L}(4.15)$ and $\operatorname{det} g=(\operatorname{det} g)(z),(4.27)$ is equivalent to

$$
v^{i} \frac{\partial}{\partial \bar{w}^{\gamma}}\left((\log G)_{\bar{\beta} ; i}(\log G)^{\bar{\beta} \alpha}\right)=(\log G)^{\bar{\beta} \alpha} v^{i} \frac{\delta_{\phi}}{\delta z^{i}}(\log G)_{\bar{\beta} \bar{\gamma}}=0,
$$

where $\frac{\delta_{\phi}}{\delta z^{i}}$ is defined by (4.1). On the other hand, by definition of $\hat{G}, \hat{P}$, and Lemma 2.2, one has

$$
\begin{aligned}
\frac{1}{G}(\bar{\partial} \hat{G})(\overline{\hat{P}}) & =\frac{\bar{v}^{l}}{G} \frac{\delta}{\delta \bar{z}^{l}}\left(G_{i j}\right) \delta v^{i} \otimes \delta v^{j} \\
& =\bar{v}^{l} \frac{\delta}{\delta \bar{z}^{l}}\left((\log G)_{i j}\right) \delta v^{i} \otimes \delta v^{j} \\
& =\bar{v}^{l} \frac{\delta \phi}{\delta \bar{z}^{l}}\left((\log G)_{i j}\right) \delta v^{i} \otimes \delta v^{j} \\
& =\bar{v}^{l} \frac{\delta_{\phi}}{\delta \bar{z}^{l}}\left(\frac{\partial^{2} \log G}{\partial w^{\alpha} \partial w^{\beta}} \frac{\partial w^{\alpha}}{\partial v^{i}} \frac{\partial w^{\beta}}{\partial v^{j}}+\frac{\partial \log G}{\partial w^{\alpha}} \frac{\partial^{2} w^{\alpha}}{\partial v^{i} \partial v^{j}}\right) \delta v^{i} \otimes \delta v^{j} \\
& =\bar{v}^{l} \frac{\delta \phi}{\delta \bar{z}^{l}}\left((\log G)_{\alpha \beta}\right) \delta w^{\alpha} \otimes \delta w^{\beta},
\end{aligned}
$$

where the third equality holds since $q_{*}\left(\frac{\delta}{\delta \bar{z}^{l}}\right)=\frac{\delta_{\phi}}{\delta \bar{z}^{l}}$. 
Combining (4.28) and (4.29), $\left\langle\left(1+\square^{\prime}\right)^{-1} i_{\bar{\partial} V} \frac{\delta}{\delta z^{i}} u^{i}, i_{\bar{\partial} V} \frac{\delta}{\delta z^{j}} u^{j}\right\rangle=0$ if and only if the complex Finsler metric $G$ satisfies (2.27). The curvature term $\Theta^{i \bar{j}}{ }_{i \bar{j}}$ in (4.20) is

$$
\Theta_{i \bar{j}}^{i \bar{j}}=-\Theta_{k \bar{l} i \bar{j}} h^{k \bar{j}} h^{i \bar{l}}=-\hat{s}_{h} .
$$

Combining (4.20) and (4.26) with (4.30), one obtains

Proposition 4.3 Let $G$ be a strongly pseudoconvex complex Finsler metric on complex manifold $M$. There exist Hermitian metrics $h$ and $g$ on $M$ such that the sum of two scalar curvatures satisfies

$$
s_{h}+\hat{s}_{h} \leq \frac{n+1}{2} \int_{\mathcal{X}_{z}} K_{G} \frac{\operatorname{det} g}{\operatorname{det} G} \frac{(\sqrt{-1} \partial \bar{\partial} \log G)^{n-1}}{(n-1) !} .
$$

Moreover, the equality holds if and only if the Finsler metric satisfies (2.27).

Let

$$
\omega_{h}=\sqrt{-1} h_{i \bar{j}} d z^{i} \wedge d \bar{z}^{j}
$$

be the fundamental form associated with the Hermitian metric $h=\left(h_{i \bar{j}}\right)$ on $M$. The first application of (4.3) is as follows.

Corollary 4.4 If $K_{G} \leq 0$ and $M$ is compact, then there exists a Hermitian metric $h$ such that

$$
\int_{M} \hat{s}_{h} \omega_{h}^{n} \leq 0
$$

Proof There is a relation between the two scalar curvatures of a Hermitian metric (see eg. [34, Formula (3.3)])

$$
s_{h}=\hat{s}_{h}+\left\langle\bar{\partial} \bar{\partial}^{*} \omega_{h}, \omega_{h}\right\rangle .
$$

Integrating both sides of above equation, one gets

$$
\int_{M} \hat{s}_{h} \omega_{h}^{n} \leq \int_{M} \hat{s}_{h} \omega_{h}^{n}+\int_{M}\left|\bar{\partial}^{*} \omega_{h}\right|^{2} \omega_{h}^{n}=\int_{M} s_{h} \omega_{h}^{n} .
$$

Combining Proposition 4.3 with the assumption $K_{G} \leq 0$, one has

$$
\begin{aligned}
\int_{M} \hat{s}_{h} \omega_{h}^{n} & \leq \frac{1}{2} \int_{M}\left(\hat{s}_{h}+s_{h}\right) \omega_{h}^{n} \\
& \leq \frac{n+1}{4} \int_{P(T M)} K_{G} \frac{\operatorname{det} g}{\operatorname{det} G} \frac{(\sqrt{-1} \partial \bar{\partial} \log G)^{n-1}}{(n-1) !} \wedge \omega_{h}^{n} \leq 0 .
\end{aligned}
$$


Theorem 4.5 Let $(M, G)$ be a strongly pseudoconvex complex Finsler metric which satisfies (2.27). If $K_{G} \geq 0$ and $K_{G}(p)>0$ for some $p \in P(T M)$, then the Kodaira dimension $\kappa(M)=-\infty$, i.e., $H^{0}\left(M, l K_{M}\right)=0$ for $l \geq 1$.

Proof By Proposition 4.3 and (2.27), so there exist Hermitian metrics $h$ and $g$ on $M$ such that

$$
s_{h}+\hat{s}_{h}=\frac{n+1}{2} \int_{\mathcal{X}_{z}} K_{G} \frac{\operatorname{det} g}{\operatorname{det} G} \frac{(\sqrt{-1} \partial \bar{\partial} \log G)^{n-1}}{(n-1) !} .
$$

By the assumption of $K_{G}$, so $s_{h}+\hat{s}_{h}$ is also semi-positive and strict positive at some point in $M$. The same proof as in [6, Theorem 1, 34, Theorem 3.1], one has $H^{0}\left(M, l K_{M}\right)=0$ for $l \geq 1$.

Acknowledgements The author thanks Professor Kefeng Liu and Professor Huitao Feng for their suggestions on the study of holomorphic sectional curvature of complex Finsler manifolds, Professor Bo Berndtsson and Professor Xiaokui Yang for many helpful discussions, and the anonymous referee for the valuable comments which helped improve the paper.

Open Access This article is distributed under the terms of the Creative Commons Attribution 4.0 International License (http://creativecommons.org/licenses/by/4.0/), which permits unrestricted use, distribution, and reproduction in any medium, provided you give appropriate credit to the original author(s) and the source, provide a link to the Creative Commons license, and indicate if changes were made.

\section{References}

1. Abate, M., Patrizio, G.: Finsler Metrics_A Global Approach. LNM, vol. 1591. Springer, Berlin (1994)

2. Abate, M., Patrizio, G.: Holomorphic curvature of Finsler metrics and complex geodesics. J. Geometr. Anal. 6(3), 341-363 (1996)

3. Aikou, T.: On complex Finsler manifolds. Rep. Fac. Sci. Kagoshima Univ. 24, 9-25 (1991)

4. Aikou, T.: Complex Finsler geometry. In: Antonelli, P. (ed.) Hand Book of Finsler Geometry, pp. 3-79. Kluwer, New York (2003)

5. Aikou, T.: Finsler Geometry on Complex Vector Bundles. Riemann-Finsler Geometry, vol. 50, pp. 83-105. MSRI Publications, Berkeley (2004)

6. Balas, A.: On the sum of the Hermitian scalar curvatures of a compact Hermitian manifold. Math. Z. 195(2), 429-432 (1987)

7. Berndtsson, B.: Curvature of vector bundles associated to holomorphic fibrations. Ann. Math. 169, 531-560 (2009)

8. Berndtsson, B.: Positivity of direct image bundles and convexity on the space of Kähler metrics. J. Differ. Geom. 81(3), 457-482 (2009)

9. Berndtsson, B.: Strict and non strict positivity of direct image bundles. Math. Z. 269(3-4), 1201-1218 (2011)

10. Cao, J., Wong, P.M.: Finsler geometry of projectivized vector bundles. J. Math. Kyoto Univ. 43(2), 396-410 (2003)

11. Choi, Y.-J.: Positivity of direct images of fiberwise Ricci-flat metrics on Calabi-Yau fibrations. arXiv:1508.00323v4 (2017)

12. Diverio, S., Trapani, S.: Quasi-negative holomorphic sectional curvature and positivity of the canonical bundle. arXiv:1606.01381

13. Feng, H., Liu, K., Wan, X.: Chern forms of holomorphic Finsler vector bundles and some applications. Int. J. Math. 27(4), 1650030 (2016)

14. Feng, H., Liu, K., Wan, X.: Geodesic-Einstein metrics and nonlinear stabilities. arXiv:1710.10243 (2017)

15. Ichijyo, Y.: Finsler manifolds modeled on a Minkowski space. J. Math. Kyoto Univ. 16, 25-50 (1976) 
16. Kim, K., Lee, H.: Schwarz's Lemma from a Differential Geometric Viewpoint. IISc Lecture Notes Series, vol. 2. World Scientific Publishing, Bangalore (2011)

17. Kobayashi, S., Ochiai, T.: On complex manifolds with positive tangent bundles. J. Math. Soc. Jpn. 22(4), 499-525 (1970)

18. Kobayashi, S.: Negative vector bundles and complex Finsler structures. Nagoya Math. J. 57, 153-166 (1975)

19. Kobayashi, S.: Hyperbolic Complex Spaces. Springer, Berlin (1998)

20. Liu, K., Yang, X.: Curvature of direct image sheaves of vector bundles and applications. J. Differ. Geom. 98, 117-145 (2014)

21. Liu, K., Sun, X., Yang, X.: Positivity and vanishing theorems for ample vector bundles. J. Algebraic Geom. 22(2), 303-331 (2013)

22. Omori, H.: Isometric immersions of Riemannian manifolds. J. Math. Soc. Jpn. 19, 205-214 (1967)

23. Schumacher, G.: Moduli of canonically polarized manifolds, higher order Kodaira-Spencer maps, and an analogy to Calabi-Yau manifolds. arXiv:1702.07628v1 (2017)

24. Shen, B., Shen, Y.: Schwarz lemma and Hartogs phenomenon in complex Finsler manifold. Chin. Ann. Math. 34B(3), 455-460 (2013)

25. To, W., Yeung, S.: Finsler metrics and Kobayashi hyperbolicity of the moduli spaces of canonical polarized manifolds. Ann. Math. 181, 547-586 (2015)

26. Tosatti, V., Yang, X.: An extension of a theorem of Wu-Yau. J. Differ. Geom. 107, 573-579 (2009)

27. Wong, P.-M., Wu, B.-Y.: On the holomorphic sectional curvature of complex Finsler manifolds. Houst. J. Math. 37(2), 415-433 (2011)

28. Wu, D., Yau, S.T.: Negative holomorphic curvature and positive canonical bundle. Invent. math. (2015). https://doi.org/10.1007/s00222-015-0621-9

29. Wu, D., Yau, S.T.: A remark on our paper "Negative holomorphic curvature and positive canonical bundle". Commun. Anal. Geometry 24(4), 901-912 (2016)

30. Yau, S.T.: Harmonic functions on complete Riemannian manifolds. Commun. Pure Appl. Math. 28, 201-228 (1975)

31. Yau, S.T.: A general Schwarz lemma for Kähler manifolds. Am. J. Math. 100(1), 197-203 (1978)

32. Yau, S.T.: Open problems in geometry. Proc. Symp. Pure. Math. 54(Part 2), 275-319 (1992)

33. Yau, S.T.: From Riemann and Kodaira to modern development on complex manifold. Jpn. J. Math. 11, 265-303 (2015)

34. Yang, X.: Hermitian manifolds with semi-positive holomorphic sectional curvature. Math. Res. Lett. 18, 10001-10023 (2015) 